\title{
RESEARCH OF URGENT BIOCHEMISTRY TEST ORDERING HABIT
}

\author{
Kubilay Elmac1 ${ }^{1}$, Betül İnce ${ }^{1}$, Sevgi Eskiocak ${ }^{2}$, Eray Özgün ${ }^{2}$
}

${ }^{1}$ Trakya University School of Medicine, Edirne, TURKEY

${ }^{2}$ Department of Medical Biochemistry, Trakya University School of Medicine, Edirne, TURKEY

\section{ABSTRACT}

Aims: This study aims to reveal the inappropriate use of biochemical laboratory testing at Trakya University Hospital Biochemistry Laboratory, increase the awareness of the physicians and prevent time loss.

Methods: This study was descriptive, retrospective and carried out by scanning data resources. Two 48-hour intervals were chosen to evaluate the test ordering habits of the physicians working at Trakya University Hospital. Between the dates of 3rd - 5th of November 2017, Trakya University Hospital Biochemistry Laboratory was working fully functionally. However, between the dates of 10th - 12th of November 2017, the automation system of the hospital was scheduled to be shutdown due to a technical error. All the physicians working at Trakya University Hospital were informed about the technical error of the automation system and were told that they would need to order only stat tests by using old-fashioned test request forms. The data of ordered tests in these two-time intervals were analyzed and compared by using frequencies and percentages as descriptive statistics.

Results: The mean number of tests per patient was 23 between 3rd - 5th of November 2017 and 15.5 between 10th - 12th of November 2017. The number of patients who had at least one test order decreased only $13.1 \%$ between 10th - 12th of November 2017. The total number of departments who made at least one test order increased by one between 10th - 12th of November 2017.

Conclusion: This study indicates that physicians should be more careful while ordering tests which are necessary. Therefore, there is a need for better communication between the laboratory staff and physicians that also plays a significant role in providing better health care for the patients.

Keywords: Biochemistry, laboratories, emergencies

\section{INTRODUCTION}

There is not a certain definition of urgent test in medical sciences. Generally, the word "stat" is being used to specify the urgent test. Stat, which comes from the Latin word statim, means immediately (1).

Laboratories have at least 2 priority levels for tests such as routine and stat. In addition, there is another priority level called as soon as possible (ASAP) that is the second most urgent category, placed between routine and stat. Volmar et al. (2) revealed that $44.2 \%$ of laboratories defined 3 priority levels that include routine, ASAP and stat in their study. Using more than 3 priority levels is not recommended due to its confusing effects on the laboratory staff (3).
In the past, the stat testing was only for the patient's convenience. However, nowadays the stat testing is being used for both the physician's and the institution's convenience besides the patient's convenience (4). This new usage of stat testing puts a heavy load on the shoulders of the laboratory staff. When laboratories cannot meet stat expectations both the physician and the laboratory workers become dissatisfied and this situation directly effects the patient's treatment (3).

Decreasing the turnaround time for stat testing is crucial for critical patients. Therefore, it is mandatory to give priority to urgent tests among thousands of tests that are ordered. To provide this priority, evidence-based use of laboratory testing by physicians carries an important role. Otherwise, piling up of stat tests extends expected 
stat turnaround times and causes delays in necessary interventions.

The aim of this study is to investigate the usage of biochemistry laboratory testing according to necessity, raise the awareness of physicians and prevent time-consuming mistakes.

\section{MATERIAL AND METHODS}

This descriptive, retrospective study was approved by Trakya University Faculty of Medicine Scientific Research Ethics Committee and carried out by scanning data resources of Trakya University Hospital Biochemistry Laboratory. In this study, biochemistry test ordering habits of the physicians in Trakya University Hospital were analyzed by comparing the amount and types of tests requested from Trakya University Hospital Biochemistry Laboratory in two consecutive weekends.

Biochemistry Laboratory of Trakya University Hospital accepts test samples from all services and policlinics of the hospital and consider the ones that are sent from emergency policlinic or defined as urgent by the physician as stat. Between the 3rd of November 2017, 18:30, and 5th of November 2017, 18:30, the laboratory was working fully functionally and this time interval was called weekend zero (W0) in the study. However, between the 10th of November 2017, 18:30 and 12th of November 2017, 18:30, the automation system of the hospital, that allows physicians to order tests online and review the results, was scheduled to be shutdown due to a technical error and this time interval was called as weekend one (W1) in the study.

All the physicians working at Trakya University Hospital were informed about the technical error of the automation system and they were requested to order only stat tests by using old-fashioned test request forms. The reason why they were requested to order only stat tests was to avoid any mistakes due to huge amount of test request forms circling around the laboratory. To determine the tests that were stat among the tests ordered in W1, the study by Sucov et al. (5) that was based on guidelines of American College of Emergency Physicians was used. All of the tests that are listed in Table 1 were considered as stat.
Table 1: Biochemistry tests that were considered as stat.

\begin{tabular}{|l|l|}
\hline \multicolumn{2}{|c|}{ Stat Tests } \\
\hline Activated partial thromboplastin time & D-Dimer \\
\hline Ammoniac & Glucose \\
\hline Amylase & N-terminal pro b type natriuretic peptide \\
\hline Arterial blood gas & Prothrombin time \\
\hline Beta human chorionic gonadotropin hormone & Serum chlorine level \\
\hline Blood urea nitrogen & Serum potassium level \\
\hline Carbon dioxide & Serum sodium level \\
\hline Complete blood count & Troponin I \\
\hline Complete urine examination & Valproic acid \\
\hline Creatinine & \\
\hline
\end{tabular}

After W0 and W1, data of all the ordered biochemistry tests in $\mathrm{W} 0$ and $\mathrm{W} 1$ were obtained from the data processing unit of the laboratory. All the patients who were taking inpatient treatment in the hospital or brought to the emergency service of the hospital were included in the study if their physician ordered at least one biochemistry test for them. The data were classified by the demographical data of patients, the time when the test was ordered, the department where the test was ordered from and the name of the test. The results of $\mathrm{W} 0$ and $\mathrm{W} 1$ were compared by using arithmetic mean, frequencies and percentages as descriptive statistics.

\section{RESULTS}

The total number of ordered tests in $\mathrm{W} 0$ and $\mathrm{W} 1$ were shown in Table 2. The mean number of tests per patient was 23 in W0, 15.5 in $\mathrm{W} 1$; the decrease was $32.6 \%$. There were 5832 stat and 4477 non stat test orders in W0, 3510 stat and 2549 non stat test orders in $\mathrm{W} 1$. The percentage of ordered tests that were accepted as stat (shown in Table 1) to total was 56.6 in W0 and 58 in $\mathrm{W} 1$. The number of patients decreased only $13.1 \%$, from 449 to 390 . The total number of departments increased by one in $\mathrm{W} 1$, from 35 to 36 . The percentage of ordered tests that were sent from the emergency department was 21.1 in W0 and 19.2 in W1.

Table 2: Total number of ordered tests in W0 and W1.

\begin{tabular}{|l|c|c|}
\hline & W0 & W1 \\
\hline Total Number of Ordered Tests (n) & 10309 & 6059 \\
\hline Number of Patients (n) & 449 & 390 \\
\hline Number of Different Types of Tests (n) & 102 & 88 \\
\hline Number of Departments (n) & 35 & 36 \\
\hline
\end{tabular}

${ }^{*}$ W0: 3rd of November 201718.30 - 5th of November 2017 18:30,

${ }^{*}$ W1: 10th of November 201718.30 - 12th of November 2017 18:30 
The most ordered non-stat tests in W0 and W1 were shown in Table 3 . There was a decrease in the orders of all nine tests, the test order that decreased the most was total bilirubin by $57.3 \%$, and the least decreased was serum aspartate aminotransferase (AST) by $30 \%$. There were 4 non-stat test orders that increased in W1 (Table 4). The most ordered stat tests were shown in Table 5.

\section{Table 3: The most ordered non stat tests in W0 and} W1.

\begin{tabular}{|l|c|c|}
\hline & \multicolumn{2}{|c|}{ Number of Ordered Tests (n) } \\
\hline Type of Test & W0 & W1 \\
\hline AST (SGOT) & 502 & 351 \\
\hline ALT (SGPT) & 501 & 348 \\
\hline CRP & 491 & 329 \\
\hline Albumin & 410 & 259 \\
\hline Total Protein & 364 & 227 \\
\hline Total Bilirubin & 295 & 126 \\
\hline Direct Bilirubin & 292 & 128 \\
\hline
\end{tabular}

AST: Aspartate aminotransferase, SGOT: Serum glutamic oxaloacetic transaminase, ALT: Alanine aminotransferase, SGPT: Serum glutamic pyruvic transaminase, CRP: C-Reactive protein, LDH: Lactate dehydrogenase, CK: Creatine kinase, ALP: Alkaline phosphatase, W0: 3rd of November 2017 18:30 - 5th of November 2017 18:30, W1: 10th of November 2017 18:30 - 12th of November 2017 18:30

Table 4: Increased non stat tests in W1.

\begin{tabular}{|l|c|c|}
\hline & \multicolumn{2}{|c|}{ Number of Ordered Tests (n) } \\
\hline Type of Test & Wo & W1 \\
\hline HDL & 9 & 13 \\
\hline LDL & 9 & 13 \\
\hline Triglyceride & 9 & 12 \\
\hline Cholesterol & 8 & 12 \\
\hline
\end{tabular}

HDL: High-density lipoprotein, LDL: Low-density lipoprotein, W0: 3rd of November 2017 18:30 - 5th of November 2017 18:30, W1: 10th of November 2017 18:30 - 12th of November 2017 18:30

\section{Table 5: The most ordered stat tests in W0 and W1.}

\begin{tabular}{|l|c|l|c|}
\hline & Number of Ordered Tests $(\mathbf{n})$ & & Number of Ordered Tests (n) \\
\hline Type of Test & W0 & Type of Test & W1 \\
\hline Complete Blood Count & 702 & Complete Blood Count & 446 \\
\hline Serum Potassium Level & 682 & Blood Urea Nitrogen & 421 \\
\hline Serum Sodium Level & 682 & Creatinine & 419 \\
\hline Serum Chlorine Level & 655 & Glucose & 313 \\
\hline Creatinine & 633 & Serum Sodium Level & 303 \\
\hline
\end{tabular}

\section{DISCUSSION}

In this study, it was apparent that every ordered test in W1 had the possibility to be a stat test. It substantially depended on the patient's current condition. However, reaching every physician and patient to check the necessity of the ordered tests, was time-consuming and nearly impossible because of the missing data due to the technical error in the automation system of the hospital. That is why it was decided to form a list that contains the most probable stat tests to make a general comparison between W0 and W1.

It was hoped that physicians would only order stat tests in $\mathrm{W} 1$ otherwise it could be very challenging for both patients and staff of the hospital. Test results had to be delayed because of the excessive amount of test orders which were logged into machines manually in W1 and this extra workload affected the patients in critical condition negatively.

The proportion of the stat tests to total is $56.6 \%$ in W0 and 58\% in W1. Since doctors were notified about the situation of the laboratory and asked to order only the stat tests, it was expected to see the ratio of stat tests much higher in W1. However, the results were not compatible with our hypothesis. The decrease in the ordered non-stat tests in W1 was ranging between $30 \%$ and $57.3 \%$ (Table 3 ). In fact, there were two possible tests that could be considered as stat in Table 3: creatine kinase (CK) and $\mathrm{C}$-reactive protein (CRP). However, some conditions stated in Guideline on Pathology Testing in the Emergency Department had to be met to consider these tests as stat (6). There were 4 different conditions that CRP testing had to be considered as stat: atraumatic back pain, fever for investigation, fever for investigation with significant travel history and suspected septic joint. Besides that, there were two conditions where CK testing had to be considered/performed as stat; snake bite and significant overdose (6). Except for CK and CRP, other tests in Table 3 could have been ordered when the laboratory turned to its usual working. This way, the extra workload of the laboratory could have been eased and actual stat tests could have been resulted on time. There was an increase in non-stat tests that were shown in Table 4 in W1 despite the situation.

Volmar et al. (2) found that $16.3 \%$ of 52 participating biochemistry laboratories could not meet 
the expected turnaround times of stat tests in their study in 2009. Andrew Sucov et al. (5) recorded a 25\% decrease in test orders in the Emergency Department of Rochester University Hospital after reporting a newly prepared guideline, without any restriction, for ordering tests to physicians in between the years 1995 and 1997. Yilmaz et al. (7) observed a decrease, ranging between $12.6 \%$ and $85 \%$ in ordered tests in Ankara Numune Training and Research Hospital after only reorganizing the test ordering screen and this also saved 371,183 USD in one year. Ordered tests per patient were 15.8 at the beginning of their study (7). In our study, ordered tests per patient was 15.5 despite the fact that the laboratory asked to physicians to send only stat tests in $\mathrm{W} 1$.

The major limiting factor of this study was missing data of W1 due to ordering tests with old-fashioned test request form. This problem prevented us from making detailed analysis such as including departments to the comparison. In addition, to evaluate the use of biochemistry laboratory tests a more detailed study that includes tracking all patients' treatments and physicians' reasons for ordering tests needs to be carried out.

In conclusion, this study shows that physicians should be more careful while ordering tests which are necessary. Therefore, there is a need for better communication between the laboratory staff and physicians which also plays a significant role in providing better health care for the patients.

Ethics Committee Approval: This study was approved by Scientific Researches Committee of Trakya University School of Medicine.

Informed Consent: Written informed consent was obtained from the participants of this study.

Conflict of Interest: The authors declared no conflict of interest.

Author contributions: Concept: KE, Bİ, SE, EÖ. Design: KE, Bİ, SE, EÖ. Supervision: KE, Bİ, SE, EÖ. Resources: KE, Bİ, SE, EÖ. Materials: KE, Bİ, SE, EÖ. Data collection and/or processing: KE, Bİ, SE, EÖ. Analysis and/or Interpretation: KE, Bİ, SE, EÖ. Literature Search: KE, Bİ, SE, EÖ. Writing Manuscript: KE, Bİ, SE, EÖ. Critical Review: KE, Bİ, SE, EÖ.

Financial disclosure: The authors declared that this study received no financial support.

Editor-in-chief's Note: One of the authors of this article, Kubilay Elmac1 is a member of the editorial board of
Turkish Medical Student Journal. However, he did not take place in any stage on the editorial decision of the manuscript. The editors who evaluated this manuscript are from another institutions.

\section{REFERENCES}

1. Tiersten D. The "stat" problem. Clin Lab Med 1983;3(3):499-507.

2. Volmar KE, Wilkinson DS, Wagar EA et al. Utilization of stat test priority in the clinical laboratory. Arch Pathol Lab Med 2013;137(2):220-7.

3. Cathcart P, Emancipator K. Preventing overuse of stat testing. Lab Med 1997;28(3):165-6.

4. Hilborne L, Lee H, Cathcart P. STAT testing? A guideline for meeting clinician turnaround time requirements. Practice parameter. Am J Clin Pathol 1996;105(6):671-5.

5. Sucov A, Bazarian JJ, deLahunta et al. Test ordering guidelines can alter ordering patterns in an academic emergency department. J Emerg Med 1999;17(3):3917.

6. Australasian College for Emergency Medicine, The Royal College of Pathologists of Australasia. Guideline on pathology testing in the emergency medicine. 2012. Available from URL: https:/www.rcpa.edu.au/getattachment/8424e33b-8688-4c7d-b5ab-fbc4c38bccb9/ Pathology-Testing-in-the-Emergency-Department (11.09.2018).

7. Yllmaz FM, Kahveci R, Aksoy A et al. Impact of laboratory test use strategies in a Turkish hospital. PLoS ONE 2016;11(4):e0153693. 